ApJ Letters, in press

\title{
Is There a Difference in Luminosity between Field and Cluster RR Lyrae Variables?
}

\author{
M. Catelan \\ NASA/Goddard Space Flight Center \\ Laboratory for Astronomy and Solar Physics, Code 681 \\ Greenbelt, MD 20771 \\ e-mail: catelan@stars.gsfc.nasa.gov
}

\begin{abstract}
Recent Hipparcos results have lent support to the idea that RR Lyrae variables in the halo field and in globular clusters differ in luminosity by $\approx 0.2$ mag. In this Letter, we study the pulsation properties of RR Lyraes in clusters with distances determined via main-sequence fitting to Hipparcos parallaxes for field subdwarfs, and compare them with the properties of field variables also analyzed with Hipparcos. We show that the period-temperature distributions for field and cluster variables are essentially indistinguishable, thus suggesting that there is no significant difference in luminosity between them.
\end{abstract}

Subject headings: Stars: horizontal-branch — stars: variables: other — Galaxy: globular clusters: individual (NGC 362, M5, M68, M15, M92)

\section{Introduction}

Accurate knowledge of the Population II distance scale is one of the most important goals in astronomy. Upon it depends, for instance, the determination of the ages of globular clusters (GC's), and thus of a firm lower limit to the age of the Universe.

RR Lyrae variables are the natural Pop. II "standard candle." Several methods have been devised to estimate their luminosities, but a consensus has not yet been reached. In particular, there appears to be a "dichotomy" between "faint" (i.e., short distance scale and old ages for the GC's) and "bright" (long distance scale and younger GC ages) calibrations. Walker (1992) and Catelan (1996) provide useful references covering the literature as of 1995. But the noted "dichotomy" has become even more clear-cut recently. Ground-based investigations have continued to appear supporting either the "short" or the "long" scale. Examples of the former include the Baade-Wesselink (e.g., Clementini et al. 1995) and statistical parallaxes (Layden et al. 1996; 
Popowski \& Gould 1998) analyses of field RR Lyraes. An example of the latter has been provided by the extensive analysis of the variables in M15 by Silbermann \& Smith (1995). The "persistent" nature of such a "dichotomy" has led some authors (e.g., VandenBerg, Bolte, \& Stetson 1996; Sweigart 1998) to speculate that there might exist a real difference in luminosity between field and cluster RR Lyrae variables. That was based in part on the (somewhat uncertain) Baade-Wesselink results of Storm, Carney, \& Latham (1994) for a few RR Lyrae variables in the GC's M5 and M92 and field counterparts of comparable metallicity.

These speculations notwithstanding, there has been widespread belief that, once the Hipparcos satellite parallax results became available, we would finally be able to decide between the "short" and "long" RR Lyrae distance scales. However, that turned out not to be the case. Based upon Hipparcos parallaxes of field subdwarfs and main-sequence fitting to GC's with well-defined deep color-magnitude diagrams, Gratton et al. (1997) and Reid (1997, 1998) have strongly claimed that the majority of the GC's in their samples are substantially farther away than previously estimated using ground-based parallaxes (but see Pont et al. 1998). Similarly, McNamara (1997b) has concluded that the Hipparcos parallaxes of field SX Phoenicis variables favor the "long" GC distance scale. These claims were supported by Hipparcos data for Cepheids (Feast \& Catchpole 1997; Madore \& Freedman 1997; see also the latest ground-based results by Laney 1998) and Miras (van Leeuwen et al. 1997), applied to the Large Magellanic Cloud (LMC). The "long" distance to the LMC is supported by the latest analysis of the SN1987a ring (Panagia, Gilmozzi, \& Kirshner 1998; but see Gould \& Uza 1998). On the other hand, Gratton (1998) has analyzed Hipparcos data for field horizontal-branch (HB) stars including three RR Lyrae variables, and found that the faint HB luminosity scale was preferred. Fernley et al. (1998) and Tsujimoto, Miyamoto, \& Yoshii (1998) have also reported, based on Hipparcos data for field RR Lyraes, luminosities which are consistent with the corresponding ground-based analyses. As argued by Gratton, the Hipparcos results thus seem to favor the existence of an intrinsic difference in luminosity (by $\approx 0.2$ mag) between $G C$ and field RR Lyraes.

However, no independent tests have thus far been applied to verify this. As is well known, $R R$ Lyrae pulsation properties depend strongly on their luminosities. The purpose of this Letter is to employ such properties to constrain the difference in luminosity between field and GC variables. Only GC's and field stars analyzed with Hipparcos will be covered. We begin in Sec. 2 by discussing the employed methods for deriving RR Lyrae temperatures. In Sec. 3, the selection criteria we have adopted are described. In Sec. 4, we demonstrate that GC variables do not show substantial period shifts with respect to field variables of similar metallicity, as opposed to what would be expected if there were an intrinsic luminosity difference between them. Finally, our results are critically discussed in Sec. 5.1

\footnotetext{
${ }^{1}$ We emphasize that the purpose of the present work is to perform a period-shift analysis at fixed temperature and metallicity. Thus, a careful analysis of the Sandage (1993) period-shift effect lies outside the scope of this Letter.
} 


\section{Estimating RR Lyrae Temperatures}

\subsection{The Carney, Storm, \& Jones (1992a) Approach}

In their Baade-Wesselink analysis of field RR Lyraes, Carney et al. (1992a, hereafter CSJ92) compiled parameters for a number of variables, including temperatures derived from near-infrared colors. Analyzing possible correlations in their database, they concluded that a simple equation exists [their eq. (16)] relating the "equilibrium temperature" $T_{\text {eq }}$, blue amplitudes $A_{B}$, pulsation

periods $P$, and metallicities $[\mathrm{Fe} / \mathrm{H}]$ for ab-type RR Lyraes. This relationship formed the basis for their discussion of the period-shift effect, and will be adopted here as a first means of estimating temperatures.

\subsection{The Catelan, Sweigart, \& Borissova (1998) Approach}

Catelan et al. (1998, hereafter CSB98) have recently reanalyzed temperatures based on the CSJ92 data. They argued that a relationship involving only $T_{\text {eq }}, A_{B}$, and $[\mathrm{Fe} / \mathrm{H}]$ would be safer to adopt in period-shift analyses than CSJ92's (Sec. 2.1), since period shifts caused by luminosity variations could easily be misinterpreted as being due to temperature variations. The idea of employing $A_{B}$ values to determine $T_{\text {eq }}$ (cf. Sandage 1981a,b) is supported by Jones et al. (1992), who state that "... at a fixed metallicity, it is likely that relative $A_{B}$ values are reliable indicators of relative temperatures."

We have rederived the CSB98 relationship for $T_{\text {eq }}$ for the same selection criteria and parameters used in our period-shift analysis of the Hipparcos sample (Sec. 3). Thus, the star SW And was also removed from the CSJ92 database, because it presents the Blazhko effect. Furthermore, the $A_{B}$ values from Blanco (1992) were adopted. (The differences are generally small, with the exception of DX Del, for which Blanco's $A_{B}$ is larger by 0.28 mag.)

Our new relationship for $\Theta_{\text {eq }}=5040 / T_{\text {eq }}$ thus reads:

$$
\Theta_{\text {eq }}=(0.868 \pm 0.014)-(0.084 \pm 0.009) A_{B}-(0.005 \pm 0.003)[\mathrm{Fe} / \mathrm{H}],
$$

with a multiple correlation coefficient $r=0.97$ and a rms deviation of $\simeq 40 \mathrm{~K}$. Fig. 1 shows that this relationship does provide a superb match to the CSJ92 equilibrium temperatures.

\section{RR Lyrae Stars: Adopted Samples}

In the present section, we lay out the selection criteria employed in our analysis. 


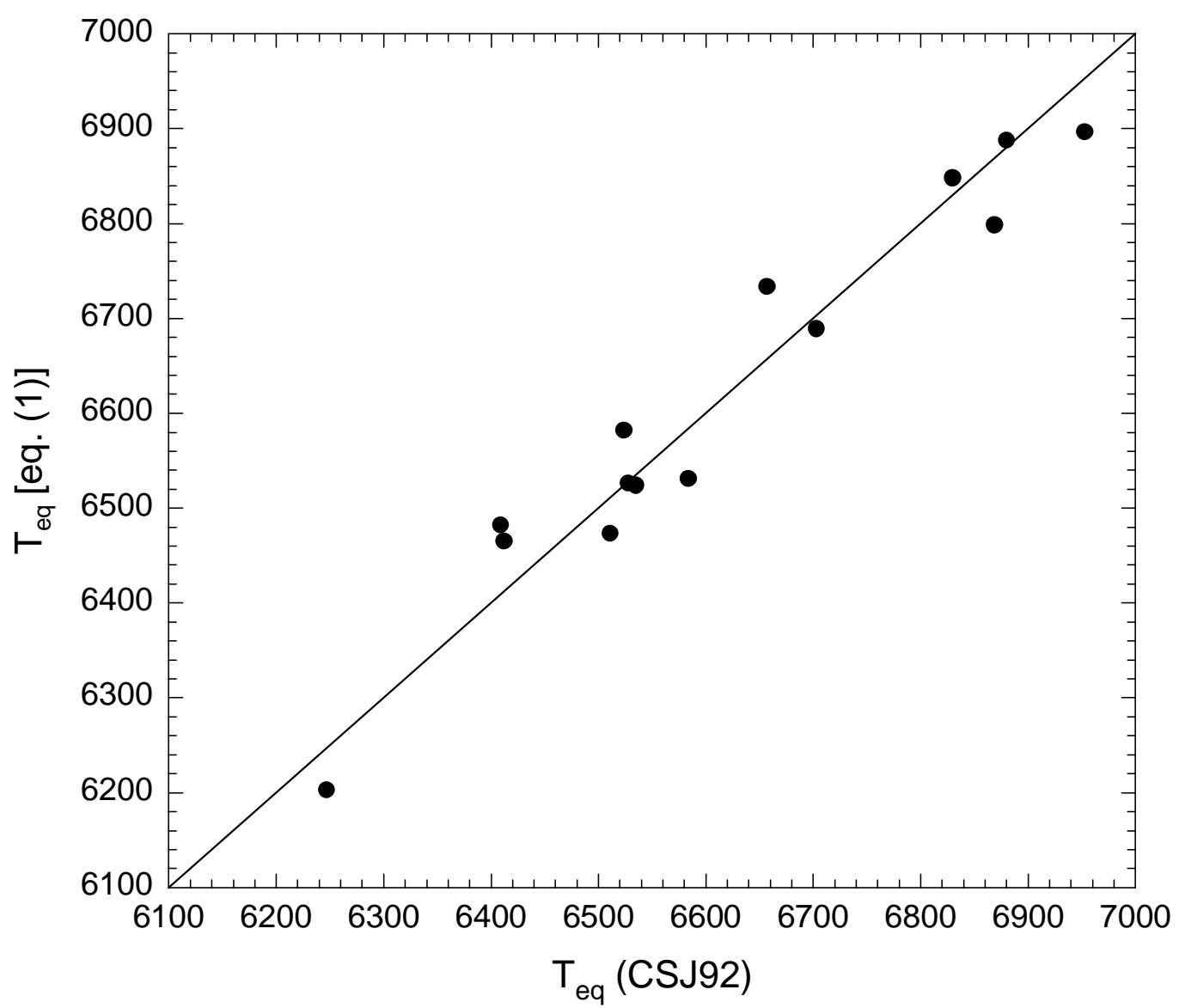

Fig. 1.- Equilibrium temperatures from eq. (1) are plotted as a function of the values actually derived by CSJ92 (and references therein).

\subsection{Field RR Lyrae Stars}

We have retrieved the list of 125 variables employed by Tsujimoto et al. (1998) in their Hipparcos-based analysis of field RR Lyraes, as kindly supplied by Dr. T. Tsujimoto.

We have selected stars from this sample according to the following criteria. i) Reliable classification as ab-type RR Lyrae stars: Variables whose RRab Lyrae nature has been questioned by Schmidt, Chab, \& Reiswig (1995) or Fernley \& Barnes (1997) were dismissed; ii) Well-behaved light curves: Stars which Blanco (1992) or Schmidt et al. pinpointed as Blazhko variables were discarded; iii) Metallicity values available from Layden: Stars for which Layden et al. (1996) do not provide metallicity values were not considered. We have also removed from the list all variables for which metallicities are based on Hemenway's (1975) measurements, since we consider the corresponding Layden et al. $[\mathrm{Fe} / \mathrm{H}]$ values quite uncertain; iv) Blue amplitudes and pulsation periods available from Blanco (1992). 
TABLE 1

RR Lyrae-"Rich" Clusters Studied with Hipparcos.

\begin{tabular}{llrrl}
\hline \hline Cluster (NGC) & Other & {$[\mathrm{Fe} / \mathrm{H}]$} & $\frac{B-R}{B+V+R}$ & References \\
\hline NGC 362 & & -1.16 & -0.87 & 1 \\
NGC 5904 & M5 & -1.29 & +0.31 & $2,3,4$ \\
NGC 4590 & M68 & -2.06 & +0.17 & 1,5 \\
NGC 7078 & M15 & -2.22 & +0.67 & 1,6 \\
NGC 6341 & M92 & -2.29 & +0.91 & 1,7 \\
\hline
\end{tabular}

Refarences.-(1) Clement 1997; (2) Brocato et al. 1996; (3) Reid 1996; (4) Storm et al. 1991; (5) Walker 1994; (6) Bingham et al. 1984; (7) Carney et al. 1992b.

\subsection{Cluster RR Lyrae Stars}

Among the 12 GC's which have had distances determined using Hipparcos parallaxes for field subdwarfs, only 5 contain a sufficiently large number of RR Lyrae variables to justify their inclusion in the present analysis: NGC 362, M5, M68, M15, and M92. Since the Layden et al. (1996) field RR Lyrae metallicities are tied in to the Zinn \& West (1984) abundance scale, we decided to adopt the $[\mathrm{Fe} / \mathrm{H}]$ entries of Harris' (1996) catalogue for consistency. Although the Zinn \& West scale has been seriously questioned by Gratton et al. (1997 and references therein), this is of minor relevance for the present purposes, since our goal is to perform a period-shift analysis at fixed metallicity. Likewise, the criticism of McNamara (1997a) of the near-infrared temperatures is of secondary relevance for us.

The adopted sources of information for the GC RR Lyrae variables are provided in Table 1, along with the cluster $[\mathrm{Fe} / \mathrm{H}]$ and Lee-Zinn HB morphology parameter (both from Harris 1996). According to such HB types, the only GC for which evolution away from the blue zero-age HB may bias the period-shift analysis is M92. As with the field star sample, Blazhko variables were discarded - as were those suspected to be non-cluster members.

\section{Period-Shift Analysis: Clusters versus Field}

Table 1 shows that our GC sample divides into two metallicity bins, with $[\mathrm{Fe} / \mathrm{H}] \approx-1.2$ and $\approx-2.2$. We have thus split the comparison between GC and field variables into two metallicity regimes. For the more metal-rich end, we employ all field RR Lyraes (25 stars) falling in the range $-1.50 \leq[\mathrm{Fe} / \mathrm{H}] \leq-0.95$ which have passed our selection criteria (Sec. 3.1); at the metal-poor end, we restrict the sample to the variables with $[\mathrm{Fe} / \mathrm{H}] \leq-1.85$ (10 stars). The resulting $\log P-\log T_{\text {eq }}$ diagrams using temperatures derived as in Sec. 2.1 and Sec. 2.2 are shown in Figs. 2 and 3, respectively.

The scatter is substantially larger in Fig. 3 than in Fig. 2. This, however, should not 
be taken as evidence that eq. (1) is less satisfactory at estimating $T_{\text {eq }}$ values than eq. (16) of CSJ92. As previously argued (Sec. 2.2), CSJ92's relationship, by including a period term, can "mask" luminosity variations at a fixed $T_{\text {eq }}$, misinterpreting them as temperature variations. Thus, eq. (16) of CSJ92 artificially drives a tight $P-T_{\text {eq }}$ distribution for a sample of stars with intrinsic luminosity scatter. Our eq. (1) does not have this bias, being more suitable for detecting luminosity variations at a given $T_{\text {eq }}$. Consider, for instance, V9 in 47 Tuc $(P=0.737 \mathrm{~d})$, which is brighter than field RR Lyraes of similar metallicity by $\approx 0.6 \mathrm{mag}$ (cf. Fig. 9 in Storm et al. 1994). Eq. (16) of CSJ92 underestimates V9's $T_{\text {eq }}$ by $\simeq 600 \mathrm{~K}$, while the underestimate from eq. (1) is only $\simeq 180 \mathrm{~K}$. In fact, Marconi's (1997, priv. comm.) pulsation models show temperatures to be quite insensitive to $L$ at fixed blue amplitude over a range in $M_{\text {bol }}$ of 0.75 mag and for $0.2 \lesssim A_{B} \lesssim 2.0$. In addition, SS Leo, which has ill-determined physical parameters, was not discarded by CSJ92 when deriving their eq. (16). Excluding this star from the CSJ92 sample, we find a reduction in the $\log P$ coefficient of their eq. (16) by $\approx$ a factor of two, and an increase (in absolute value) in the corresponding $A_{B}$ coefficient by a similar factor. The $T_{\text {eq }}$ value adopted by CSJ92 for this star, $\sim 6400 \mathrm{~K}$, differs from the one expected on the basis of eq. (1) by $\simeq 300 \mathrm{~K}-\mathrm{a}$ factor of $\approx 3$ larger than the one for the largest-deviating star in our Fig. 1.

Figs. 2 and 3 show that there is no detectable difference in period-shift properties between the studied field and cluster RR Lyraes, either at the metal-poor or at the more metal-rich end -irrespective of the approach used to estimate $T_{\text {eq }}$. If Gratton's (1998) suggestion were correct and the GC variables were brighter by $\approx 0.2 \mathrm{mag}$, we would expect to see a difference as large as $\Delta \log P \approx+0.067$ at fixed $T_{\text {eq }}$ between GC and field stars (Catelan 1996), which is most decidedly not present in our diagrams.

Other interesting conclusions that may be drawn from Figs. 2 and 3, but which we shall not discuss in the present Letter, are: i) There seems to be no offset in the $P-T_{\text {eq }}$ diagrams between GC's with widely different HB types but similar [Fe/H] (cf. Catelan 1994); ii) Metal-poor RRab Lyraes may have a cooler $T_{\text {eq }}$ cutoff than the metal-rich ones (Sandage 1993); iii) There may be an offset of $\Delta \log P \approx+0.05$ at constant $T_{\text {eq }}\left(\Rightarrow \Delta M_{\text {bol }} \approx 0.15\right.$ mag for fixed mass) between the metal-poor and the more metal-rich RR Lyraes.

\section{Discussion}

The present analysis does not substantiate Gratton's (1998) suggestion, based on Hipparcos results, that there is a difference in luminosity between $G C$ and field RR Lyrae variables, showing instead that they have essentially the same distribution in the $P-T_{\text {eq }}$ plane, both at the metal-poor and at the more metal-rich ends.

Does this imply that there is really no difference between GC and field HB stars? Not necessarily. In fact, at $[\mathrm{Fe} / \mathrm{H}]>-1$, Sweigart \& Catelan (1998) found (following the same approach as in the present Letter) substantial differences between field and (some) GC RR Lyraes 
(see also Storm et al. 1994 and Layden 1995). Moreover, it should be noted that: i) The $A_{B}-T_{\text {eq }}$ diagram (and possibly even spectroscopically-derived metallicities) may be sensitive to the helium abundance $Y$, so that an additional, $Y$-dependent term may be needed to put eq. (1) on a firmer basis (CSB98). In any case, available models suggest that it would not be possible for differences in $Y$ between field and GC stars to be consistent with both a luminosity difference of $\approx 0.2 \mathrm{mag}$ and the remarkable overlapping in the $P-T_{\text {eq }}$ plane found in Figs. 2 and 3 ; ii) As well known, field red giants do not seem to show signatures of non-canonical deep mixing, whereas some GC's do (cf. Kraft et al. 1997). It might be worth examining whether RR Lyrae temperatures and amplitudes might be sensitive to their (inherited) abundance anomalies; iii) As pointed out by Sweigart (1998), the stars which are more likely to be affected by "helium mixing" during the red giant branch phase are the blue-HB and extreme-HB stars, not the cooler RR Lyraes.

The present results (see also Fernley 1993) provide motivation for searching for systematic errors in methods employed to estimate the distances of GC's (esp. main-sequence fitting) and the luminosities of RR Lyrae stars (esp. the statistical parallaxes and Baade-Wesselink methods). Unless some "cosmic conspiracy" is leading to the remarkable agreement between field and cluster stars in Figs. 2 and 3, the "long" and "short" Pop. II distance scales cannot be reconciled in the way suggested by Gratton (1998). We cannot tell whether the "long" or the "short" scale should be preferred on the basis of a comparison with evolutionary models, due to extant systematic uncertainties in the empirical RR Lyrae temperatures (McNamara 1997a). However, the LMC provides a means of estimating RR Lyrae luminosities (Walker 1992), and several methods seem to favor the "long" distance scale (implying brighter RR Lyraes and younger GC ages) over the "short" one.

The author would like to thank C. Cacciari, A. Sweigart, D. VandenBerg, W. Landsman and the referee for useful suggestions, and M. Marconi and T. Tsujimoto for providing relevant information. This work was performed while the author held a National Research Council-NASA/GSFC Research Associateship. 


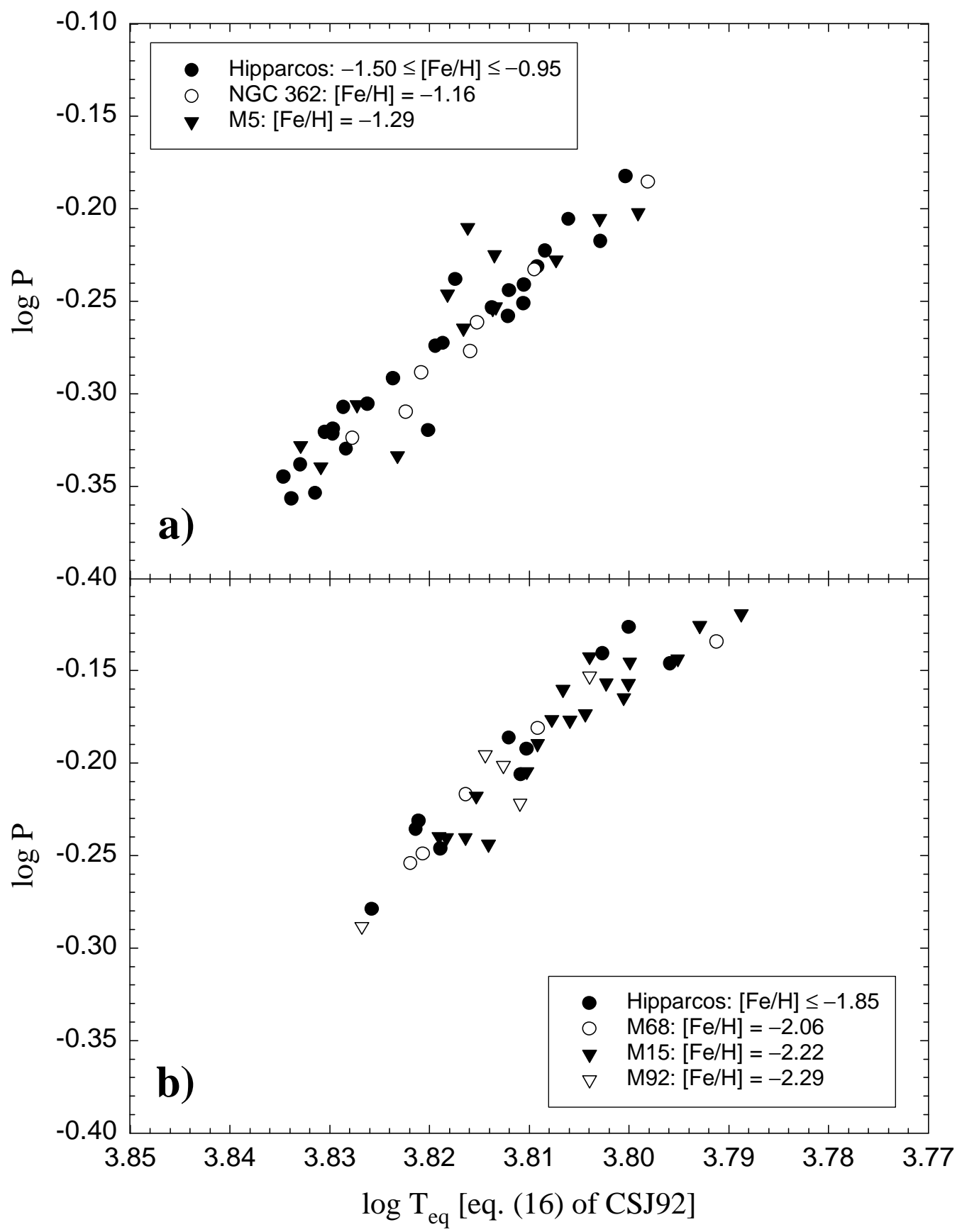

Fig. 2.- a) Period-temperature diagram for RR Lyraes in the GC's NGC 362 (o) and M5 ( $\mathbf{v}$ ), compared with variables investigated with Hipparcos $(\bullet)$ in the indicated metallicity range. b) As in panel (a), except that the more metal-poor regime is studied. Variables in M68 (o), M15 (v) and M92 $(\nabla)$ are shown. Temperatures have been derived from eq. (16) of CSJ92. 


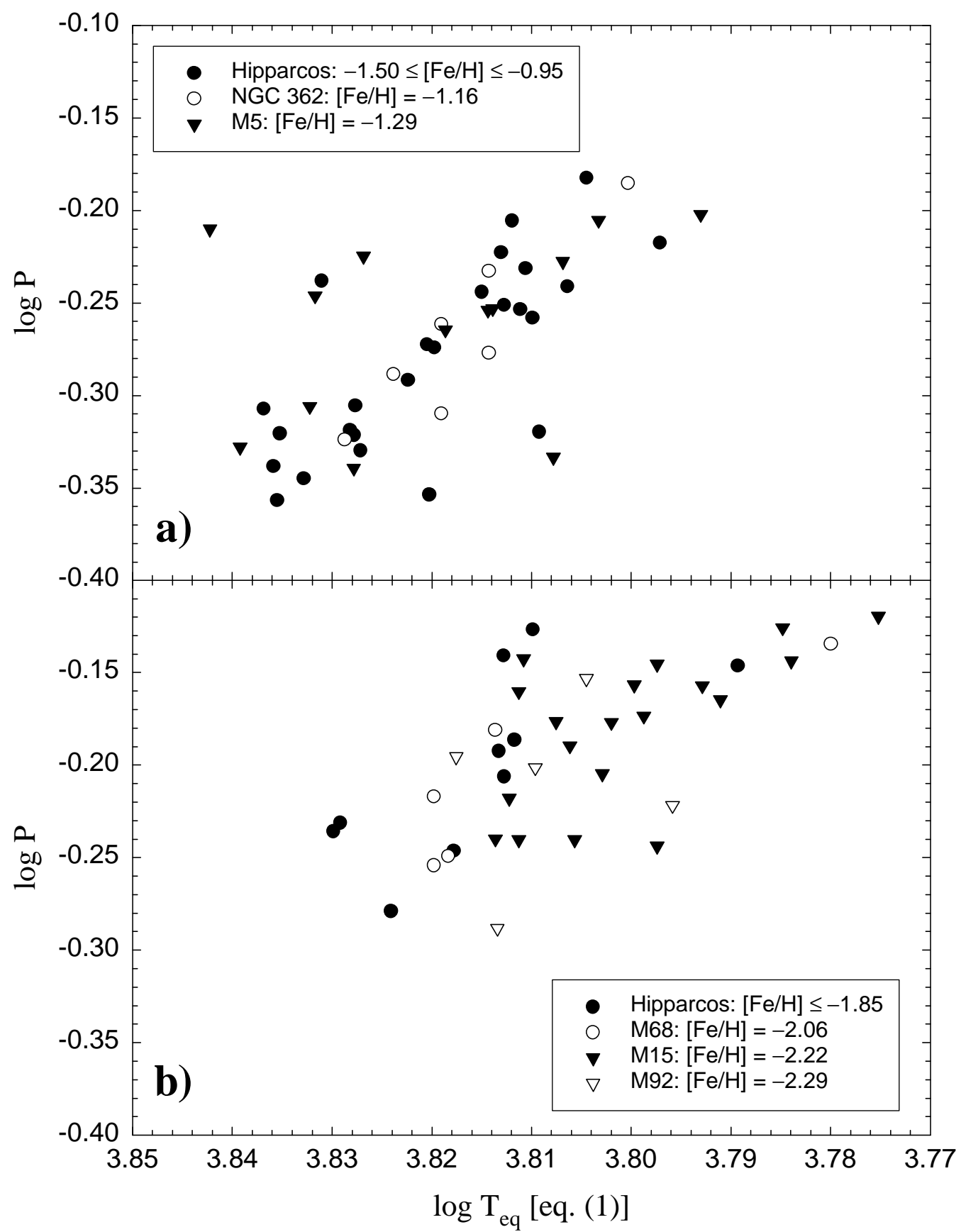

Fig. 3.- As in Fig. 2, except that temperatures have been derived from our eq. (1). 


\section{REFERENCES}

Bingham, E. A., Cacciari, C., Dickens, R. J., \& Fusi Pecci, F. 1984, MNRAS, 209, 765

Blanco, V. M. 1992, AJ, 104, 734

Brocato, E., Buonanno, R., Malakhova, Y., \& Piersimoni, A. M. 1996, A\&A, 311, 778

Carney, B. W., Storm, J., \& Jones, R. V. 1992a, ApJ, 386, 663 (CSJ92)

Carney, B. W., Storm, J., Trammell, S. R., \& Jones, R. V. 1992b, PASP, 104, 44

Catelan, M. 1994, AJ, 107, 2077

Catelan, M. 1996, A\&A, 307, L13

Catelan, M., Sweigart, A. V., \& Borissova, J. 1998, in ASP Conf. Ser. 135, A Half Century of Stellar Pulsation Interpretations: A Tribute to Arthur N. Cox, ed. P. A. Bradley \& J. A. Guzik (San Francisco: ASP), 41 (CSB98)

Clement, C. M. 1997, Helen Sawyer Hogg's Fourth Catalogue of Variable Stars in Globular Clusters, AAS Newsletter, 84, 15

Clementini, G., Carretta, E., Gratton, R., Merighi, R., Mould, J. R., \& McCarthy, J. K. 1995, AJ, 110, 2319

Feast, M. W., \& Catchpole, R. M. 1997, MNRAS, 286, L1

Fernley, J. 1993, A\&A, 268, 591

Fernley, J., \& Barnes, T. G. 1997, A\&AS, 125, 313

Fernley, J., Barnes, T. G., Skillen, I., Hawley, S. L., Hanley, C. J., Evans, D. W., Solano, E., \& Garrido, R. 1998, A\&A, 330, 515

Gould, A., \& Uza, O. 1998, ApJ, in press astro-ph/9705051)

Gratton, R. G. 1998, MNRAS, in press (astro-ph/9710271)

Gratton, R. G., Fusi Pecci, F., Carretta, E., Clementini, G., Corsi, C. E., \& Lattanzi, M. 1997, ApJ, 491, 749

Harris, W. E. 1996, AJ, 112, 1487

Hemenway, M. K. 1975, AJ, 80, 199

Jones, R. V., Carney, B. W., Storm, J., \& Latham, D. W. 1992, ApJ, 386, 646

Kraft, R. P., Sneden, C., Smith, G. H., Shetrone, M. D., Langer, G. E., \& Pilachowski, C. A. 1997, AJ, 113, 279

Laney, C. D. 1998, in ASP Conf. Ser. 135, A Half Century of Stellar Pulsation Interpretations: A Tribute to Arthur N. Cox, ed. P. A. Bradley \& J. A. Guzik (San Francisco: ASP), 180

Layden, A. C. 1995, AJ, 110, 2312

Layden, A. C., Hanson, R. B., Hawley, S. L., Klemola, A. R., \& Hanley, C. J. 1996, AJ, 112, 2110 
Madore, B. F., \& Freedman, W. L. 1997, ApJ, 492, 110

McNamara, D. H. 1997a, PASP, 109, 857

McNamara, D. H. 1997b, PASP, 109, 1221

Panagia, N., Gilmozzi, R., Kirshner, R. P. 1998, in ASP Conf. Ser., SN1987a: Ten Years After, ed. M. Phillips \& N. Suntzeff, in press

Pont, F., Mayor, M., Turon, C., \& VandenBerg, D. A. 1998, A\&A, 329, 87

Popowski, P., \& Gould, A. 1998, ApJ, in press (astro-ph/9703140)

Reid, I. N. 1996, MNRAS, 278, 367

Reid, I. N. 1997, AJ, 114, 161

Reid, I. N. 1998, AJ, in press astro-ph/9710311)

Sandage, A. 1981a, ApJ, 244, L23

Sandage, A. 1981b, ApJ, 248, 161

Sandage, A. 1993, AJ, 106, 703

Schmidt, E. G., Chab, J. R., \& Reiswig, D. E. 1995, AJ, 109, 1239; erratum: 1995, AJ, 110, 2439

Silbermann, N. A., \& Smith, H. A., 1995, AJ, 110, 704; errata: 1996, AJ, 111, 567

Storm, J., Carney, B. W., \& Beck, J. A. 1991, PASP, 103, 1264

Storm, J., Carney, B. W., \& Latham, D. W. 1994, A\&A, 290, 443

Storm, J., Nordström, B., Carney, B. W., \& Andersen, J. 1994, A\&A, 291, 121

Sweigart, A. V. 1998, in The Third Conference on Faint Blue Stars, ed. A. G. D. Philip, J. Liebert, \& R. A. Saffer (Cambridge: CUP), in press (astro-ph/9708164)

Sweigart, A. V., \& Catelan, M. 1998, in ASP Conf. Ser. 135, A Half Century of Stellar Pulsation Interpretations: A Tribute to Arthur N. Cox, ed. P. A. Bradley \& J. A. Guzik (San Francisco: ASP), 39

Tsujimoto, T., Miyamoto, M., \& Yoshii, Y. 1998, ApJ, 492, L79

van Leeuwen, F., Feast, M. W., Whitelock, P. A., \& Yudin, B. 1997, MNRAS, 287, 955

VandenBerg, D. A., Bolte, M., \& Stetson, P. B. 1996, ARA\&A, 34, 461

Walker, A. R. 1992, ApJ, 390, L81

Walker, A. R. 1994, AJ, 108, 555

Zinn, R., \& West, M. J. 1984, ApJS, 55, 45 